\title{
THE UV-FEL AT THE NSLS: STRAIGHT INJECTION CONFIGURATION
}

\author{
Xiaohao Zhang and Juan C. Gallardo \\ National Synchrotron Light Source and Physics Department \\ Brookhaven National Laboratory, Upton, New York 11973
}

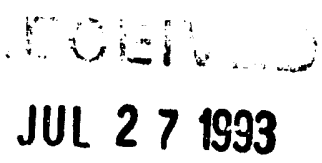

We present a design of a colinear RF-photocathode gun and linac sections for the ultraviolet free-electron laser under study at the National Synchrotron Light Source. Invorso Bolmholtz solenoid pair is used to control the divergence of the electron beam and present a slightly convergent and small beam into a conventional SLAC linac. The relative distance of the gun to the linac and the strength of the solenoidal field are chosen to minimize the emittance growth of the beam due to space charge. The simulation codes PARMELA and TRACE-3D are used to model the beam from the cathode to the entrance to the wiggler.

\section{INTRODUCTION}

The National Synchrotron Light Source (NSLS) is studying the design and construction of an ultraviolet free-electron laser (UV-FEL) capable of providing tunable coherent radiation from 3000 to $750 \mathrm{~nm}$. $[1,2]$

In this design study we use the by now well known BNL RF-photocathode gun [3] supplemented with an inverse Helmholtz solenoid pair [4] to control the divergence of a few picosecond and highly correlated electron pulse. The coils are located symmetrically with respect to the cathode to insure $B_{z}=0$ at the plane where the electrons are emitted. In the low energy section, the main cause of emittance dilution is the space charge. [5] We will show in the next section that an interplay between (linear) solenoidal focusing forces and de-focusing space charge fields lead to a minimum of the transverse emittance inside of the linac. [6]

The well known simulation codes PARMELA [7] and TRACE-3D [8] have been used as design tools.

\section{LONGITUDIN AL BEAM SLICES AND EMITTANCE CORRECTION}

To study the causes of emittance dilution in the transport and acceleration of a high brightness electron beam, it is desirable to divide the few picosecond bunch in longitudinal slices $(\leq 10)$ of equal length. We follow each slice through the transport system and observe that the intrinsic emittance is small ( $\approx 4-5 \pi \mathrm{mm}-\mathrm{mrad}$ ) but the projection in 2-D phase space of all slices gives rise to the typical fan plots. Although the area swept by each slice is relatively small, the area covengullisliges is significantly larger as is, consequently, the total emittance. It is easily seen that the space charge is greater in the core of the beam than at the ends and as a result, the core tends to have larger radius and $x^{\prime}$ than the ends which leads to ellipses in phase space of different orientation or slope with the larger slope corresponding to the core of the beam.

In Ref. [5], it was proposed ways to force the fan to close and effectively correct the emittance. Here we follow the suggestion in Ref. [6]; we use a solenoid to compensate the de-focusing forces of space charge. As discussed in Ref. [9], the optimal situation leading to a minimum of emittance corresponds to an ellipse in phase space that rotates from the first to the fourth and back again to the first quadrant with the particles in the tail with larger $x$ and $x^{\prime}$ than the corresponding coordinates of the particles in the core of the pulse. In this condition space charge alone tends to close the fan and consequently to decrease the total emittance of the bunch. At this point, if the beam is left to drift, space charge will eventually spread the fan again and restore the emittance to its previous higher value. However, in our design the waist occurs inside of the linac. As the beam is accelerated, the slice ellipses are frozen with respect to each other due to a decrease of space charge $O\left(\frac{1}{\gamma^{2}}\right)$. The final result after appropriate tuning is an emittance for the entire beam which is essentially the slice emittance. The initial parameters of the electron beam are shown in table I.

TABLE I. Parameters used in the PARMELA simulations for an axially symmetric $1 \frac{1}{2}$-cell gun in a $\pi$-mode configuration with a solenoid pair.

\begin{tabular}{lc}
\hline \hline RF frequency [MHz] & 2856 \\
Radius of aperture [cm] & 1.0 \\
Radius of nose [cm] & 1.0 \\
First cell length [cm] & 2.625 \\
Second cell length [cm] & 5.23 \\
Field on cathode [MV/m] & 100.0 \\
Initial phase [degree] & 45.0 \\
Laser spot size (Uniform top-hat profile) [cm] & 0.1 \\
Laser pulse width (Uniform top-hat profile) [ps] & 7.0 \\
Charge (2.4 nC with a chicane) [nC] & 2.5 \\
Solenoidal field Bo [kG] & 2.2 \\
Solenoid length [cm] & 15.0 \\
\hline \hline
\end{tabular}

\section{DISCLAIMER}

DISTRIEUTION OF THIS DOCUMIUNT IS This report was prepared as an account of work sponsored by an agency of the United States Government. Neither the United States Government nor any agency thereof, nor any of their employees, makes any warranty, express or implied, or assumes any legal liability or responsibility for the accuracy, completeness, or usefulness of any information, apparatus, product, or process disclosed, or represents that its use would not infringe privately owned rights. Referencet hérein to añy specific commercial product, prosess, or service by trade name, trademark, manufacturer, or otherwise does not necessarily constitute or imply its endorsement, recommendation, or favoring by the United States Government or any agency thereof. The views and opinions of authors expressed herein do not necessarily state or reflect those of the United States Government or any agency thereof. 
The emittance correction scheme just described only corrects linear forces. Therefore, a uniform laser illumination of the cathode with sharp edges is required, as only this type of distribution leads to linear space charge forces. [10,11] An emittance (rms - normalize) plot as a function of the longitudinal coordinate $z$ is shown in Fig. 1. In that figure we observe a fast increase of the emittance $\epsilon^{\text {norm }}$ occurring immediately after the gun and a subsequent reduction to a rather broad minimum (in some cases there is a gentle asymptotic decrease) located inside the first linac section. This behavior of $\epsilon^{\text {norm }}$ reflects the interplay of (linear) solenoid focusing and defocusing space charge fields and illustrates the mechanism discussed above.

\section{ELECTRON BEAM LINE DESCRIPTYON}

The beam line consists of a high brightness electron source, an accelerating and a transport section. The total length of the beam line is about 25 meters. The 2.4 $\mathrm{nC}$ electrons are produced by an RF-photocathode gun with a pair of symmetric solenoids. A length adjustable drift $(\approx 75 \mathrm{~cm})$ after the gun is followed by the first linac section which increase the beam energy from $4.0 \mathrm{MeV}$ to $70 \mathrm{MeV}$. The strength of the solenoids and the drift length between the gun and the first linac section are adjusted to have a convergence beam going into the linac and a double waist at the end of it. The initial phase of the cavity is tuned for the beam to have the correct longitudinal phase space for magnetic compression. The compression is achieved by a chicane designed to reduce the length of the electron pulse by a factor of 2 and to increase the current of the front of the pulse by a factor of 3. After compression the electron are accelerated to $360 \mathrm{MeV}$ by four additional linac sections. The beam is accelerated a slightly off the peak of the RF wave to reduce the energy spread of beam. At the entrance of each linac section, a quadrupole triplets is located to provide necessary focussing to keep the beam size small. It also provide a flexibility for later improvement and modifications.

\section{PARMELA RESULTS}

We present results with and without magnetic compression.

With magnetic compression: Fig. 2 depicts the pulse length in picosecond and rms (global) energy spread of the pulse. Fig. 3 illustrates the current of individual slices as the beam progresses through the transport line. We notice that the tail of the beam carries less than $25 \%$ of the total charge.

The characteristics of the electron beam presented to the wiggler is summarize in table II.
TABLE II. Electron beam parameters at the end of the transport line (case with a chicane) SLICES 1 AND 2

\begin{tabular}{|c|c|}
\hline Average energy $[\mathrm{MeV}]$ & 362 \\
\hline $\boldsymbol{\gamma}$ & 710 \\
\hline$Q$ first two slices ( $80 \%$ of $2.4 \mathrm{nC}$ ) [nC] & 1.88 \\
\hline Energy spread $[\%]$ & 0.14 \\
\hline Length of the pulse [ps] & 2.75 \\
\hline Current $(820 \times 0.75+276 \times 0.25)[\mathrm{A}]$ & 684.0 \\
\hline$r m s \epsilon^{\text {norm }}[\pi \mathrm{mm}-\mathrm{mrad}]$ & 6.0 \\
\hline rms radius $[\mathrm{mm}](x, y)$ & $0.8,0.4$ \\
\hline rms divergence $(x, y)$ & $-0.04,-0.025$ \\
\hline
\end{tabular}

Without magnetic compression: The electron beam parameters at the end of the transport line are in table III, notice we are retaining only $90 \%$ of the $2.5 \mathrm{nC}$.

In a work under preparation, we will include errors, such as solenoids misalignment, stability of the coil power supply and wake fields in the linac.

TABLE III. Electron beam parameters at the end of the transport line

\begin{tabular}{lc}
\hline \hline Average energy [MeV] & 326 \\
$\gamma$ & 640 \\
rms Global energy spread [\%] & 0.3 \\
rms Local energy spread [\%] & 0.06 \\
rms length of the pulse [ps] & 3.1 \\
Current $\left(I=\frac{Q}{\left.\sqrt{2 \pi \sigma_{2}^{m-}}\right)}\right.$ [A] & 290 \\
rms $\epsilon^{\text {norm }[\pi \text { mm-mrad] }}$ & 5.7 \\
$\alpha(x, y)$ & $4.7,4.2$ \\
$\beta$ [cm] $\left(x, y^{\prime}\right)$ & $6.4,6.4$ \\
$r m s$ radiug [rnm] $(x, y)$ & $0.85,0.8$ \\
$r m s$ divergence $(x, y)$ & $0.06,0.05$ \\
\hline \hline
\end{tabular}

\section{SUMMARY}

We have presented the design of a high-brightness injection line for the proposed UV-FEL at the NSLS, Brookhaven National Laboratory. The study of slices of the electron pulse is essential to tune the compensating effects of the solenoid (focusing) and space-charge forces (de-focusing) as emittance correction scheme. Furthermore, magnetic compression leads to a pulse with $\approx 685 . A$, global energy spread of 0.14 and normalized emittance of $\approx 6.0 \pi \mathrm{mm}-\mathrm{mrad}$.

\section{ACKNOWLEDGEMENTS}

The authors wish to thanks B. Palmer and H. Kirk for illuminating discussions. We also acknowledge the active support and encouragement of I. Ben-Zvi. This research was supported by the U.S. Department of Energy under Contract No. DE-ACO2-76-CH00016. 
FIG. 2. a) History of the rms pulse length in picosecond without compression.

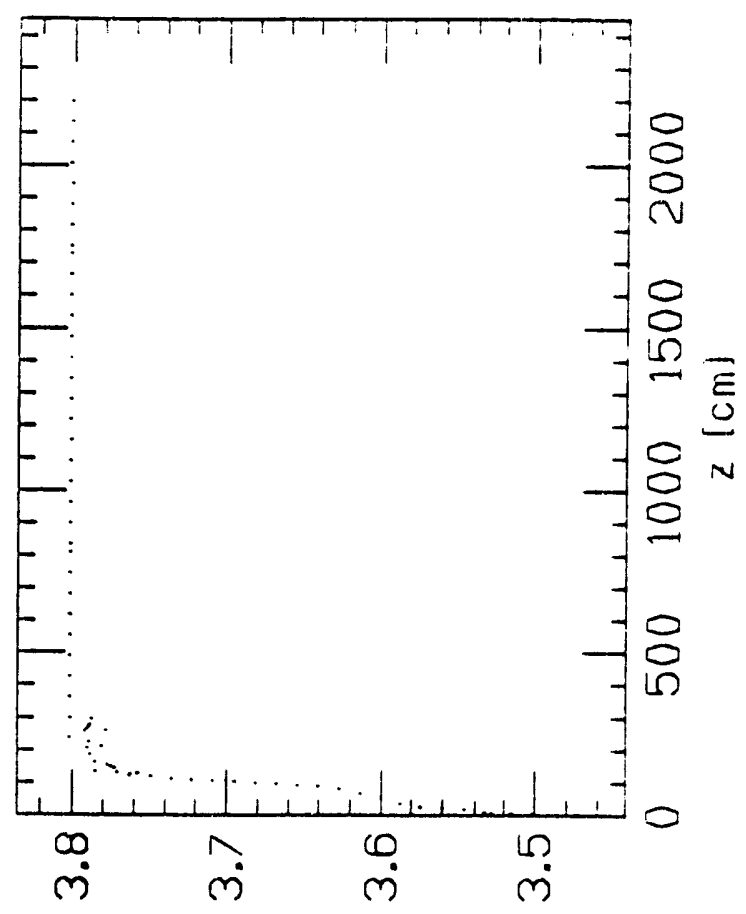

1] UV Free-Electron Laser. Conceptual Design Report, BNL Report No. 48565, February (1993).

[2] X. Zhang, Juan C. Gallardo, Low energy beam transport in the NSLS UV-FEL, BNL report, unpublished.

[3] K. Batchelor et al., Nucl. [nstr. and Meth., A318, 372 (1992)

[4] R. L. Sheffield, AIP Conference Proceedings, 184, 1502 (1990) and reference there in; K. McDonald, unpublished; Zhi-Yu Jiang and C. Pellegrini, private communication.

[5] Juan C. Gallardo and Robert B. Palmer, Nucl. Instr. and Meth., A304, 345 (1991)

[6] R. L. Sheffield et al., Nucl. Instr. and Meth., A318, 282 (1992) and references there in.

[7] L. Young, private communication.

[8] K. R. Crandall, TRACE-3D, LA-UR-90-4146 Los Alamos Accelerator Code Group, December (1990).

[9] Juan C. Gallardo and H. Kirk, An injection scheme for the Brookhaven ATF utilizing space-charge emittance growth compensation, this Proceedings.

[10] S. Humphries, Jr., Nucl. Instr. and Meth., A258, 548 (1987)

[11] Juan C. Gallardo, Control of non-linear space-charge emittance growth, BNL-43862 (1990)

FIG. 1. History of the rms normalised transverse emittance $\epsilon^{\text {nown }}(x, y)$ vs. distance $z$ without compression.

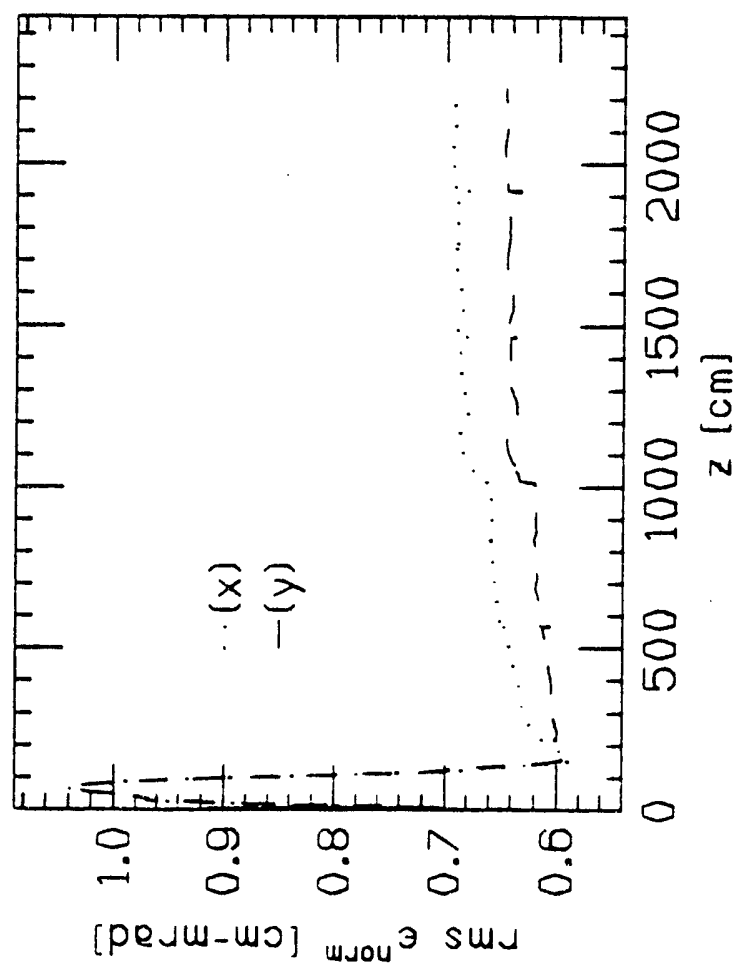

FIG. 3. History of the current of 5 slices along the transport line without compression.

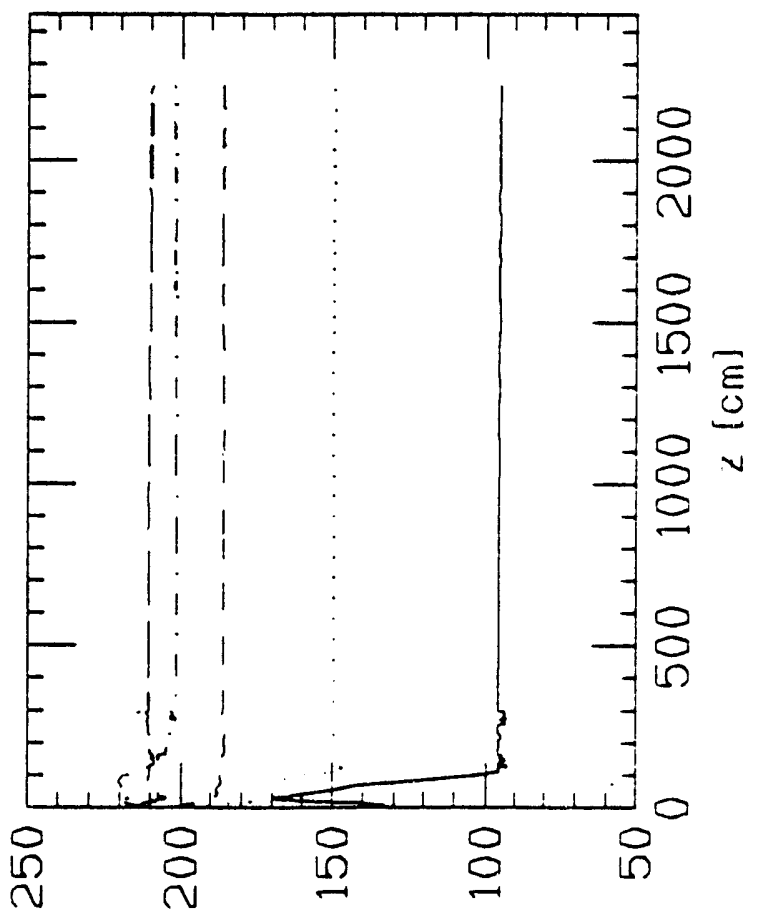

$[\forall]$ I 

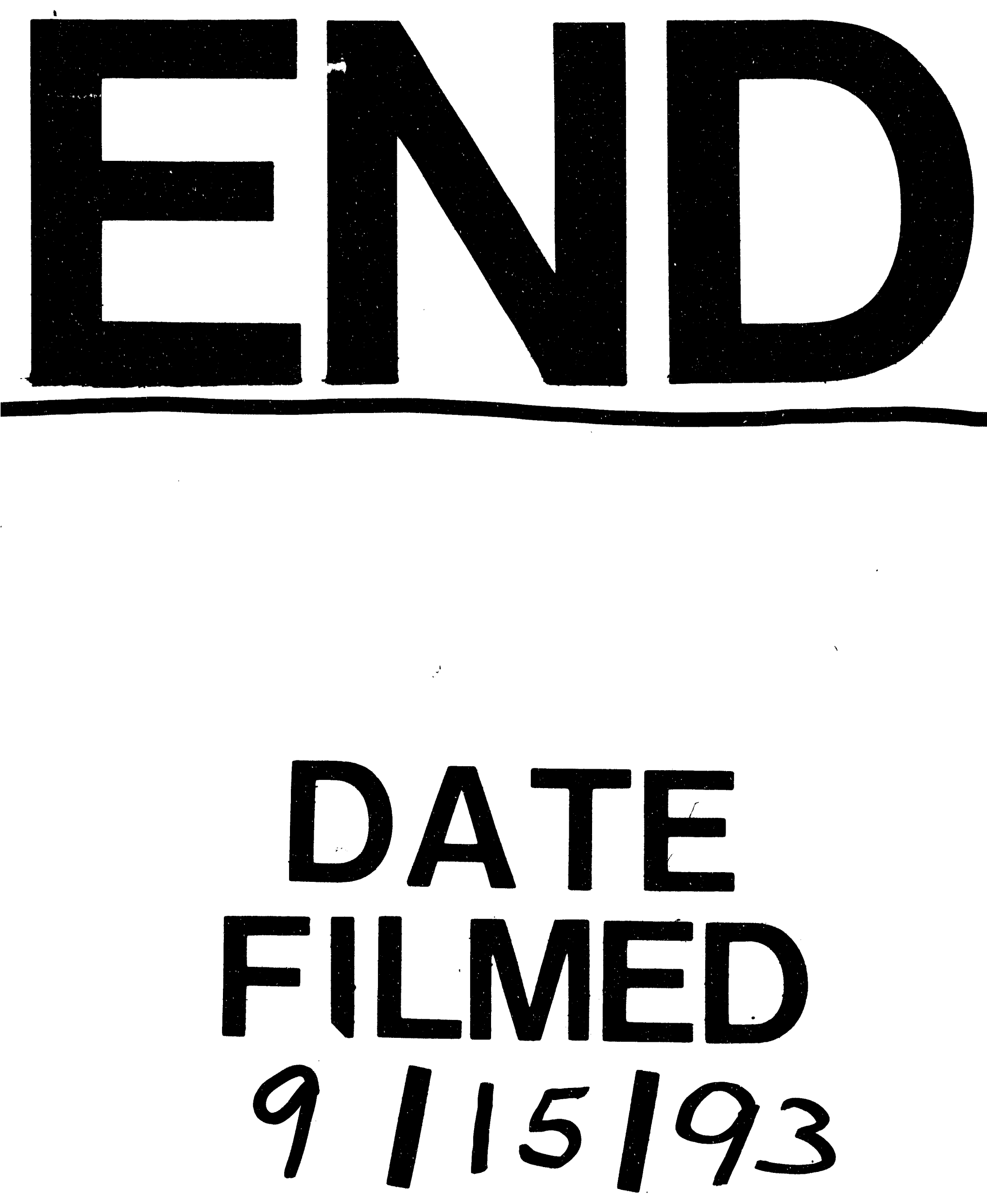

I 
